\title{
Structural characterization of amphiphilic siderophores produced by a soda lake isolate, Halomonas sp. SL01, reveals cysteine-, phenylalanine- and proline-containing head groups
}

\section{Authors: Luis O'mar Figueroa, Benjamin Schwarz, \& Abgail M. Richards}

NOTICE: The final publication is available at Springer via http://dx.doi.org/10.1007/s00792-015-0790-x.

Figueroa LO, Schwarz B, Richards AM, "Structural characterization of amphiphilic siderophores produced by a soda lake isolate, Halomonas sp. SL01, reveals cysteine-, phenylalanine- and prolinecontaining head groups," Extremophiles Nov 2015 19(6):1183-1192.] 


\section{Structural characterization of amphiphilic siderophores produced by a soda lake isolate, Halomonas sp. SL01, reveals cysteine-, phenylalanine- and proline-containing head groups}

\section{Luis O'mar Serrano Figueroa ${ }^{1,4} \cdot$ Benjamin Schwarz ${ }^{2}$. Abigail M. Richards 3,4}

1 Department of Microbiology and Immunology, Montana State University, Bozeman, MT 59717, USA

2 Department of Chemistry and Biochemistry, Montana State University, Bozeman, MT 59717, USA

3 Department of Chemical and Biological Engineering, Montana State University, Bozeman, MT 59717,

4 USA forter for Biofilm Engineering, Montana State University, Bozeman, MT 59717, USA

Abstract Soap Lake, located in Washington State, is a naturally occurring saline and alkaline lake. Several organ-isms inhabiting this lake have been identified as producers of siderophores that are unique in structure. Bacterial iso-lates, enriched from Soap Lake sediment and water sam-ples, were screened for siderophore production using both the chrome azurol S (CAS) agar plate and liquid methods. Bacterial isolate Halomonas sp. SL01 was found to produce relatively high concentrations of siderophores in liquid medium (up to $40 \mu \mathrm{M}$ ). Siderophores from the isolate were separated from the culture supernatant using solid phase extraction and purified by high-performance liquid chromatography (HPLC). Siderophore structure was determined using LC/MS/MS (liquid chromatography/mass spectrome-try/mass spectrometry) and fatty acid methyl ester (FAME) GC. Two distinct new families of amphiphilic siderophores were produced by isolate SL01. All siderophores ranged in size from 989 to 1096 atomic mass units and consisted of a conserved peptidic head group (per family), which coor-dinates iron, coupled to fatty acid moieties. The fatty acyl moieties were $\mathrm{C} 10 \mathrm{â}$ " $^{\text {"C }} \mathrm{C} 14$ in length and some with hydroxyl substitutions at the third $\hat{\mathrm{I}} \pm$ position. These siderophores resembled amphiphilic aquachelin siderophores produced by Halomonas aquamarina strain DS40M3, a marine bac-terium as well as siderophores from isolate Halomonas sp. SL28 that was found to produce amphiphilic siderophores.
Bacteria thriving under saline and alkaline conditions are capable of producing unique siderophores resembling those produced by microbes inhabiting marine environments.

Keywords Halomonas - Amphiphilic siderophores - Cysteine - Phenylalanine - Proline Halochelins

\section{Introduction}

Iron is an important element for different biological pro-cesses in most microorganisms, with the exception of some lactobacilli (Crosa 2004). Siderophores are low molecu-lar weight entities with high iron (III) affinity. These mol-ecules help the microbial cell to obtain iron (III) due to its low bioavailability $\left(10^{-18} \mathrm{M}\right)$ at a nearly neutral $\mathrm{pH}$ envi-ronment (Raymond and Dertz 2004). As different micro-organisms are found in any type of environment, there are different siderophore types as well. Siderophores can be classified into three categories by the functional groups involved in the iron coordination process: hydroxamates, catecholates and carboxylate. The acylation of ornithine produces hydroxamic acid groups permitting hydroxamate siderophores to coordinate iron; meanwhile, catechol and its molecular modifications, such as like 2,3-dihydroxy benzoic. 
acid, are required for the catecholate siderophores to coordinate iron; carboxylate siderophore must have $\alpha$-hydroxy donor groups (Butler and Theisen 2010; Pattus and Abdallah 2000). Some of siderophores will possess only one of the classes of iron-coordinating groups while others may utilize multiple ones. For example, aerobactin, produced by $E$. coli, contains both hydroxamic and hydroxycarboxylic coordinating groups (Gauglitz et al. 2012; Valdebenito et al. 2006). Amphiphilic siderophores are still another class of iron-chelating molecules that are set apart by the combination of a polar amino acid head group and an aliphatic fatty acid tail attached to the $\mathrm{N}$-terminus of the head group, lending amphiphilic characteristics. Like non-amphiphilic siderophores, amphiphilic siderophores may contain a variety of iron-coordinating moieties such as catechols, $\alpha$-hydroxycarboxylic acid and hydroxamic acids within the polar head group. Citrate and hydroxyaspartic acid have also been found as part of amphiphilic siderophores structure (Sandy and Butler 2009). Examples of the variability found in functional groups of amphiphilic siderophores are ochrobactins (Martin et al. 2006), synechobactins (Ito and Butler 2005) and petrobactins (Homann et al. 2009a).

To date, most of the amphiphilic siderophores come from marine microorganisms, such as Marinobacter sp. and Vibrio sp. (Amin et al. 2012; Butler and Theisen 2010; Sandy and Butler 2009; Sandy et al. 2010). In this report, we describe two distinct families of amphiphilic siderophores, both produced by an isolate from Soap Lake located in Washington State, Halomonas sp. SL01. Soap Lake is a meromictic lake with a $\mathrm{pH}$ of 9.8 and a dissolved solid concentration ranging from $140 \mathrm{~g} / \mathrm{L}$ in the monimolimnion layer to $14 \mathrm{~g} / \mathrm{L}$ in the mixolimnion layer (Edmondson and Anderson 1965; Sorokin et al. 2007). The microbial population in Soap Lake is diverse and includes phylogenetic groups $\alpha-, \beta-$, and $\gamma$-Proteobacteria, Acidobacteria, Verrucomicrobia, Synechococcus, Actinobacteria and Thermotogales, among others (Dirnitriu et al. 2008). Also, Fe(III) reduction has been characterized on different isolates including Bacillus sp. (Pollock et al. 2007) and our siderophore-producing halophile Halomonas sp. SL01 (VanEngelen et al. 2008). It is implied then that this reduction process is due to siderophore production by this microorganism. The main objective of this report is to determine if the hypersaline bacterial isolate, Halomonas sp. SL01, produces siderophores and if so, to identify the chemical structure of the molecule or molecules.

\section{Methods}

\section{Growth medium}

The growth media used in all the experiments were an arti- where Halomonas sp. SL01 was isolated. The media were prepared with $50.0 \mathrm{~g} / \mathrm{L}$ sodium chloride (Fisher), $1.12 \mathrm{~g} / \mathrm{L}$ sodium borate, $1.0 \mathrm{~g} / \mathrm{L}$ ammonium chloride, $0.06 \mathrm{~g} / \mathrm{L}$ calcium chloride, $0.05 \mathrm{~g} / \mathrm{L}$ magnesium chloride hexahydrate, $0.85 \mathrm{~g} / \mathrm{L}$ sodium nitrate, $0.50 \mathrm{~g} / \mathrm{L}$ potassium phosphate monobasic, $0.01 \mathrm{~g} / \mathrm{L}$ potassium chloride, $0.25 \mathrm{~g} / \mathrm{L}$ yeast extract and sodium pyruvate $(5.0 \mathrm{~g} / \mathrm{L}$, Fisher) as the carbon source. The media were treated with Chelex (Sigma Aldrich) resin according to the manufacturer's instructions to reduce iron and glassware was acid washed. All media were filter sterilized with $0.22 \mu \mathrm{m}$ polyethylene sulfonate (PES, Nalgene) membrane filters and $\mathrm{pH}$ was adjusted to 9.0 with $10 \mathrm{~N} \mathrm{NaOH}$ (Fisher).

\section{Siderophore detection in solution by CAS assay}

Stock solutions were prepared in advance: (1) $10 \mathrm{mM}$ HDTMA (Fisher); (2) iron solution: $1 \mathrm{mM} \mathrm{FeCl}{ }_{3} \bullet \mathrm{H}_{2} \mathrm{O}$ (Acros) in $10 \mathrm{mM} \mathrm{HCl}$; (3) $2 \mathrm{mM}$ aqueous chrome azurol sulfonate (CAS, Sigma) and (4) 0.2 M 5-sulfosalicylic acid (Fisher). Then, the CAS assay solution was prepared by adding $6 \mathrm{~mL}$ of $10 \mathrm{mM}$ HDTMA in a volumetric flask $(100 \mathrm{~mL})$ diluted with a bit of water. In that same flask, $1.5 \mathrm{~mL}$ iron solution and $7.5 \mathrm{~mL}$ of $2 \mathrm{mM}$ CAS solution were added. Then, in a separate flask, $4.307 \mathrm{~g}$ anhydrous piperazine (Acros) was dissolved in some water and $6.25 \mathrm{~mL}$ of $12 \mathrm{M} \mathrm{HCl}$ was added. This buffer was rinsed into the volumetric flask with HDTMA, CAS and iron solution and volume completed with nanopure water.

To determine the presence and concentration of siderophores in solution, a $500 \mu \mathrm{L}$ aliquot of media supernatant (previously centrifuged at $12,000 \times \mathrm{g}$ for $5 \mathrm{~min}$, Eppendorf 5417C) plus $500 \mu \mathrm{L}$ CAS assay solution were added to a cuvette. Then, $20 \mu \mathrm{L}$ of 5 -sulfosalicylic acid (or to a final concentration of $4 \mathrm{mM}$ ) was added. Absorbance of the solution at $630 \mathrm{~nm}$ was measured after equilibrium was reached ( $30 \mathrm{~min}-2 \mathrm{~h}$, but no longer than $6 \mathrm{~h}$ ). Concentrations were determined based on ferrioxamine B standard curve.

\section{Growth culture preparation and sampling}

To prepare the growth cultures, a frozen stock of Halomonas sp. SL01 was inoculated in $100 \mathrm{~mL}$ SLM. The sterile control was $50 \mathrm{~mL}$ of SLM. Starter culture and control were placed in the shaker incubator (140 rpm at room temperature or $37^{\circ} \mathrm{C}$; Infors HT Ecotron) and optical densities (OD, at $600 \mathrm{~nm}$ ) and CAS assays (at $630 \mathrm{~nm}$ ) were done once a day withdrawing $1 \mathrm{~mL}$ aliquots. Once the CAS absorbance read about $0.100,1 \mathrm{~mL}$ aliquots were transferred into 3, $1 \mathrm{~L}$ baffled flasks with $400 \mathrm{~mL}$ SLM. A sterile control flask was also prepared for the study. Flasks were incubated at previous conditions and OD and CAS readings were taken twice a day until a maximum siderophore 
production was detected. Finally, the media were ultra-centrifuged (Sorvall Instruments RC5C; GSA rotor) at $6238 \times \mathrm{g}$ for $20 \mathrm{~min}$ at $4^{\circ} \mathrm{C}$.

\section{SLM and CAS plates}

To check for culture purity, SLM plates were streaked. To prepare the plates, Noble agar $(30 \mathrm{~g} / \mathrm{L}$, Difco) was dissolved and sterilized by autoclaving in $500 \mathrm{~mL}$ nanopure water; the solution was placed in a water bath at $60^{\circ} \mathrm{C}$. Soap Lake media were prepared as previously mentioned, $\mathrm{pH}$ adjusted and filter sterilized with $0.22 \mu \mathrm{m}$ membrane filter and placed in a water bath for $3 \mathrm{~h}$. When solutions were at temperature, $500 \mathrm{~mL}$ of SLM was poured in the Noble agar and allowed to mix by magnetic stirring. Plates were poured and solidified overnight. When growth culture was in stationary phase and siderophore production was detected, streaks of SLM plates were done.

CAS plates were prepared in a similar way to SLM plates. A CAS/HDTMA mix was prepared by first mixing chrome azurol sulfonate $(605 \mathrm{mg})$, water $(500 \mathrm{~mL})$ and $1 \mathrm{mM} \mathrm{FeCl}{ }_{3}$ in $10 \mathrm{mM} \mathrm{HCl}(100 \mathrm{~mL})$ together (Solution A). HDTMA (729 mg) was dissolved in water $(400 \mathrm{~mL}$, Solution B). Solution A was added to B, providing gentle stirring. The mixture was sterilized and placed in a water bath. Noble agar solution was prepared as previously mentioned and sterilized (placed in water bath). SLM ( $400 \mathrm{~mL}$ ) was filter sterilized and placed in a water bath. SLM and CAS/HDTMA solutions were mixed with the Noble agar solution and plates were poured and solidified overnight. Streak plates were done as a double verification for siderophore production and culture purity.

\section{Siderophore extraction, purification and lyophilization}

A C2 column (Varian) was used to remove siderophores from cell-free supernatant. Each column was conditioned with methanol (Fisher) and nanopure water according to the manufacturer's instructions. To extract siderophores from the supernatant, a $50 \mathrm{~mL}$ volume was run through the column. After loading the column with culture supernatant, the column was filled with nanopure water and this phase was collected. After this, the column was filled with methanol; this phase was collected in a second tube. Then, the column was filled with a second portion of nanopure water and this was eluted and discarded. This process was repeated until all supernatant had passed through the column. All methanol extracts and first collected nanopure water were pooled. Extracts were concentrated by evaporation centrifugation (at $50^{\circ} \mathrm{C}$, Labconco) for $2 \mathrm{~h}$, stored at $4{ }^{\circ} \mathrm{C}$ and purified by HPLC (Dionex).

To purify the extracts obtained from Halomonas sp. SL01, an HPLC method was created. Briefly, two mobile phases were used: (A) water with $0.01 \%$ (v/v) trifluoroacetic acid (TFA, Fisher) and (B) acetonitrile (Fisher) with $0.01 \%$ (v/v) TFA. The mobile phase gradient was $10-70 \%$ (v/v) B for $63 \mathrm{~min}$. A C4 reverse phase column (4.6 mm ID X $250 \mathrm{~mm}$, Grace) was used. UV detection wavelength was set to $220 \mathrm{~nm}$. Siderophore fractions were collected, frozen, lyophilized overnight and stored $\left(4{ }^{\circ} \mathrm{C}\right)$ for future tests.

\section{Mass spectrometry (MS) analysis}

To confirm siderophore presence, lyophilized samples were dissolved in nanopure water and the CAS assay was performed. Siderophore-positive samples were analyzed on an Agilent 6538 QTOF LC/MS with electro spray ionization (ESI) to determine their chemical structure. Samples were further purified using a 10-100 \% (v/v) acetonitrile gradient for $10 \mathrm{~min}$ on the LC before entering the MS. Acidified water with $0.1 \%$ (v/v) formic acid was used as aqueous buffer. Samples were analyzed in positive mode and with a fragmentation voltage of $150 \mathrm{~V}$. MS/MS analysis was done on the same equipment with a constant stream of directly infused sample administered with a syringe pump. A target ion was selected from the MS analysis and fragmentation voltage was ramped in cycle to provide a progression of fragments for each sample (Tables 2, 3). Data analysis was done using the Bruker's DataAnalysis software.

\section{Fatty acid methyl ester (FAME)}

To determine aliphatic tail structure, fatty acid methyl ester (FAME) analyses were done by MIDI Labs, Inc. Lyophilized siderophore samples were dissolved in nanopure water and analyzed through FAME in a four-step reaction process: (1) saponification, (2) methylation, (3) extraction and (4) wash for sample clean up. Four reagents were prepared to help cleaved the tail from the siderophore and they were particularly related to each reaction step in FAME. Reagent 1 (for saponification) was made from $45 \mathrm{~g}$ $\mathrm{NaOH}, 150 \mathrm{~mL}$ methanol and $150 \mathrm{~mL}$ distilled water. Reagent 2 (for methylation) was made with $325 \mathrm{~mL}$ certified $6.0 \mathrm{~N} \mathrm{HCl}$ and $275 \mathrm{~mL}$ methyl alcohol. The fatty acid was poorly soluble in the aqueous phase at this point. Reagent 3 (for extraction) was made of $200 \mathrm{~mL}$ hexane and $200 \mathrm{~mL}$ methyl tert-butyl ether. This reagent extracted the fatty acid tails into the organic phase for use with the gas chromatograph (GC). Reagent 4 (for sample clean up) was made of $10.0 \mathrm{~g} \mathrm{NaOH}$ dissolved in $900 \mathrm{~mL}$ distilled water.

Sample processing to prepare GC ready extracts was made following the 4 steps mentioned previously. Briefly, for saponification, $1 \mathrm{~mL}$ of reagent 1 was added to the siderophore samples. Tubes were sealed and vortexed (5-10 s) and heated in a boiling water bath for $5 \mathrm{~min}$, at which time the tubes were vortexed again and placed in 
the bath for an additional $25 \mathrm{~min}$. In the methylation reaction step, $2 \mathrm{~mL}$ of reagent 2 was added. The tubes were capped and vortexed and tubes were heated for $10 \pm 1 \mathrm{~min}$ at $80 \pm 1{ }^{\circ} \mathrm{C}$ and after this samples were cooled at room temperature. For the extraction step, $1.25 \mathrm{~mL}$ of reagent 3 was added, tubes recapped and tumbled in a clinical rotator for $10 \mathrm{~min}$. The aqueous phase was discarded. To clean up the sample, about $3 \mathrm{~mL}$ of reagent 4 was added to the organic phase; the tubes were tumbled for $5 \mathrm{~min}$. After that, approximately $2 / 3$ of the organic phase was then analyzed in the GC.

\section{Gas chromatography (GC)}

After FAME, samples were analyzed in a gas chromatograph (Agilent Technologies 5890, 6890 and 6850) with Sherlock MIS Software. A $25 \mathrm{~mm} \times 0.2 \mathrm{~mm}$ phenyl methyl silicone fused silica capillary column (Ultra 2) was used. The method increased temperature from $170{ }^{\circ} \mathrm{C}$ to $270{ }^{\circ} \mathrm{C}$ at $5{ }^{\circ} \mathrm{C} / \mathrm{min}$. To ensure column cleaning and life span, a $300{ }^{\circ} \mathrm{C}$ ballistic temperature increase was held for 2 min after each sample was analyzed. A flame ionization detector was also employed to provide good sensitivity. Hydrogen was the carrier gas, nitrogen the "make up" gas and air was used to support the flame. GC calibration was done using a standard with mixtures of straight chain saturated fatty acids from 9 to 20 carbons in length (9:0-20:0) and 5 hydroxy acids.

\section{Results}

\section{Siderophore production in soap lake media and purification}

Strain SL01 from the genus Halomonas sp. grew very well in Soap Lake media. The conditions to which it was subjected were, as previously mentioned, $5.0 \%(w / v) ~ \mathrm{NaCl}$ concentration, room temperature $\left(25^{\circ} \mathrm{C}\right)$ and $\mathrm{pH} 9.0$. As shown in Fig. 1, a lag phase was absent. In contrast, a delay was detected for siderophore production. Production of siderophores detected by the chrome azurol sulfonate (CAS) assay was stable after $76 \mathrm{~h}$ of growth and harvesting was done for eventual purification. A maximum siderophore production was detected $(38.1 \mu \mathrm{M})$. Optical density reached a maximum of 0.625 , decreased and increased again up to 0.530 .

Purification of the lipid extract of the media via reverse phase chromatography yielded at least 19 unique features (Online Resource 1). Peaks B, C, D, E and F yielded ironchelating species by CAS analysis and were considered candidate siderophores. Peaks $\mathrm{A}$ and $\mathrm{H}$ were also collected and examined but were not active for iron chelation while

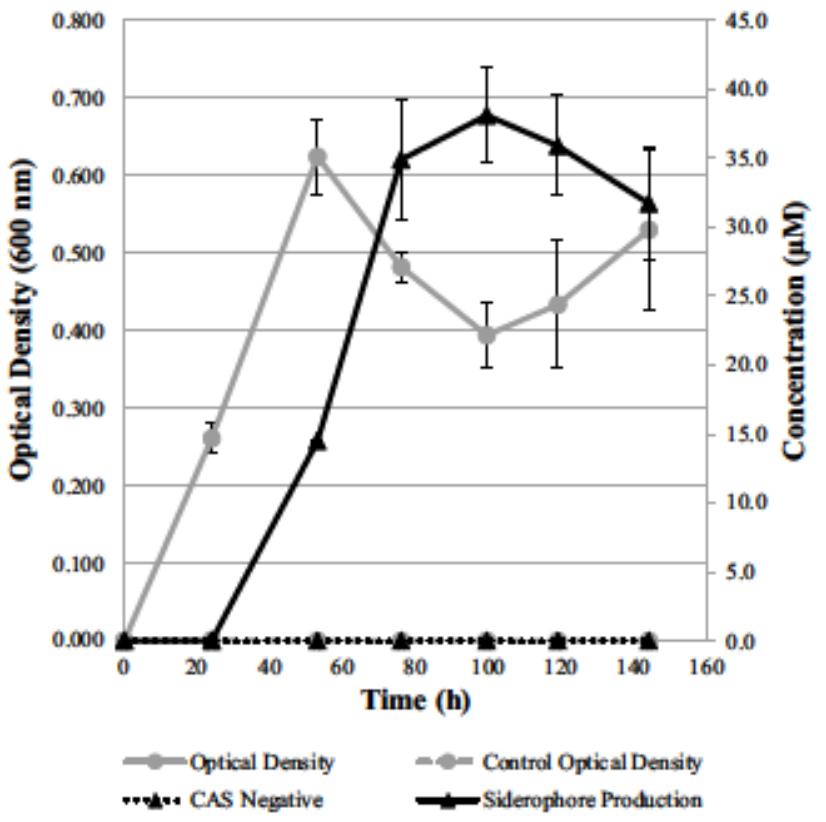

Fig. 1 Growth and siderophore production of Halomonas sp. SL01at $5 \% \mathrm{NaCl}$ soap lake media and room temperature conditions. Gray circles with solid line represent growth in terms of optical density; gray circles with dashed line represent optical density control; black triangles with solid line represent siderophore concentration in solution; and black triangles with dotted line represent siderophore concentration negative control. Time, in hours, is on the $x$ axis, optical density is on the primary $y$ axis and concentration is on the secondary $y$ axis. Values are average $\pm S D$

peak $\mathrm{G}$ was not soluble in water and could not be analyzed. Table 1 presents a summary of the iron-chelating activities of the corresponding peaks revealing siderophore presence in B-F. Fractions active in iron chelation were further analyzed to determine siderophore structure via mass spectrometry and fatty acid methyl ester.

\section{Structural analysis of siderophores by mass spectrometry and fatty acid methyl ester (FAME)}

To study siderophore structure, a QTOF LC/MS tandem MS/MS method was developed. Initial results showed two different siderophore families produced by Halomonas sp. SL01. For all siderophore samples, both a "y" series, breaking at the peptide bond and including the c-terminus, and a "b" series, breaking at the peptide bond and including the N-terminus, were observed in the fragmentation spectra. Three siderophore fractions (B, C and E) have nearly identical " $y$ " fragmentation patterns. This sharing of fragments suggested a common amino acid composition in the head group. The " $b$ " fragmentation series within this group included the same peak spacing but was shifted by a different constant mass for each sample, suggesting that the distinguishing feature is the length and/or structure of 
Table 1 HPLC fractions with siderophore detection and absence as per the CAS assay

\begin{tabular}{lll}
\hline Fraction & $\begin{array}{l}\text { Siderophore presence by CAS } \\
\text { assay }^{\mathrm{a}}\end{array}$ & $\begin{array}{l}\text { Apparent mass } \\
(\mathrm{amu})^{\mathrm{b}}\end{array}$ \\
\hline A & - & - \\
B & + & 1052.45 \\
C & + & 1096.45 \\
D & + & 989.4 \\
E & + & 1080.48 \\
F & + & 1017.38 \\
G & - & - \\
H & - & - \\
Control & - & - \\
\hline
\end{tabular}

"Symbols " + " and "-"means siderophore detection and absence, respectively

b Symbol "-_"means that fractions were not analyzed in mass spectrometry due to negative CAS assay result (no siderophore detected)

the hydrophobic tail attached at the N-terminus. Fractions $\mathrm{D}$ and $\mathrm{F}$ also shared a common " $\mathrm{y}$ " fragmentation series, while the "b" series was distinguished by a constant mass shift again suggesting common amino acid sequence in the head group and differing tails. The fragmentation patterns in both the " $y$ " and "b" series were almost entirely unique between the D and F set and the B, C \& E set. This suggests that Halomonas sp. SL01 produces distinct two families of amphiphilic siderophores for which we propose the name, halochelins.

For Halochelins B, C and E, molecular weights were calculated to be 1091.39 atomic mass units (amu), 1135.42 amu and $1119.43 \mathrm{amu}$, respectively (Online Resources 2 through 4, respectively). Parent ions showed adducts to potassium $(\mathrm{M}+\mathrm{K})$ providing the apparent total mass for each molecule (refer to Table 1). The corresponding amino acid residue sequence for the polar head group of these molecules was found to be ( $\mathrm{N}$ - to $\mathrm{C}$-terminus): phenylalanine (Phe), threonine (Thr), cysteine (Cys), arginine (Arg), glutamine (Gln), threo- $\beta$-OH-asp (Thr- $\beta-\mathrm{OH}$-asp) and $\mathrm{D}-\mathrm{N}-\mathrm{OH}$-ornithine (D-N-OH-orn). Accounting for the constant mass shift in the "b" series, these fragments also confirmed the proposed amino acid sequence. Table 2 shows a summary of fragmentation found with mass spectrometry studies.

An anticipated molecular difference in the hydrophobic tail could be calculated from the constant mass shifts in the "b" series fragments within a family. Halochelin B total apparent mass was $1052 \mathrm{amu}$ and Halochelin $\mathrm{C}$ mass was $1096 \mathrm{amu}$. The mass difference between them was $44 \mathrm{amu}$, which suggested an additional $\left(-\mathrm{CH}_{2} \mathrm{CH}_{2} \mathrm{OH}-\right)$ group in Halochelin $\mathrm{C}$ presumably in the hydrophobic tail. By comparing Halochelins $\mathrm{B}$ and E, a 28 amu difference was found. This suggested an additional $\left(-\mathrm{CH}_{2} \mathrm{CH}_{2}-\right)$
Table 2 Mass spectrometry pattern fragmentation for Halochelins B, $\mathrm{C}$ and $\mathrm{E}$ produced by Halomonas sp. SL01

\begin{tabular}{llll}
\hline $\begin{array}{l}\text { Fragments } \\
\text { (C- to N-terminus) } \\
\text { y (amu) }\end{array}$ & $\begin{array}{l}\text { B } \\
\text { b (amu) }\end{array}$ & $\begin{array}{l}\text { C } \\
\text { b (amu) }\end{array}$ & $\begin{array}{l}\text { E } \\
\text { b (amu) }\end{array}$ \\
\hline $\begin{array}{l}\text { Halochelins } \\
131\end{array}$ & 921 & 965 & 949 \\
262 & 790 & 834 & 818 \\
390 & 662 & 706 & 690 \\
546 & 506 & 550 & 534 \\
649 & 403 & 447 & 431 \\
750 & 302 & 346 & 330 \\
897 & 155 & 199 & 183 \\
\hline
\end{tabular}

" $y$ " fragments break at the peptide bond and including the c-terminus. " $b$ " series fragments break the peptide bond and include the n-terminus

group for Halochelin E. A 16 amu difference was found between Halochelins $\mathrm{C}$ and $\mathrm{E}$ that suggests to an additional hydroxyl group $(-\mathrm{OH})$ for the former. The structural groups described above were associated with fatty acid tail differences for the respective siderophores. One fragment, which was observed at low abundance, for each siderophore also suggested the approximate molecular mass for the fatty acid tails: $155 \mathrm{amu}$ for Halochelin B, 199 amu for Halochelin C and 183 amu for Halochelin E (Table 2). For more detailed information about fragments generated, please refer to Online Resources 2 through 4.

Fatty acid methyl ester (FAME) analysis was used via gas chromatography (GC) to confirm fatty acid tail presence and structure in each siderophore. Various fatty acid GC fractions were present in the lyophilized sample for Halochelins B and C (analyzed in the same fraction). At retention times, 1.2238 and $2.0545 \mathrm{~min}$ GC fractions were identified by the Sherlock MIS Software to be related to Halochelins B and C, respectively (Online Resource 5). Halochelin B fatty acid fraction (retention time $1.2238 \mathrm{~min}$ ) revealed a fatty acid with structure 10:0, suggesting that the aliphatic tail was composed of a 10 carbon chain with no double bonds or side groups (Online Resource 6). Halochelin $\mathrm{C}$ showed a fatty acid fraction (retention time $2.0545 \mathrm{~min}$ ) with a 12:0 $3 \mathrm{OH}$ structure, which suggested a 12 carbon chain, no double bonds and a hydroxyl $(-\mathrm{OH})$ side group on the third $\alpha$ carbon position. The difference in atomic mass from FAME revealed an additional 44 amu to Halochelin C that correlated with MS data. Halochelin E HPLC lyophilized fraction was also analyzed and the GC showed, at a retention time $1.6482 \mathrm{~min}$, a fatty acid with structure 12:0 (Online Resources 7 and 8). This suggested a 12-carbon chain, with no double bonds or side group substitutions. The atomic mass difference between Halochelins E and B was 28 amu, correlating with the findings of MS 

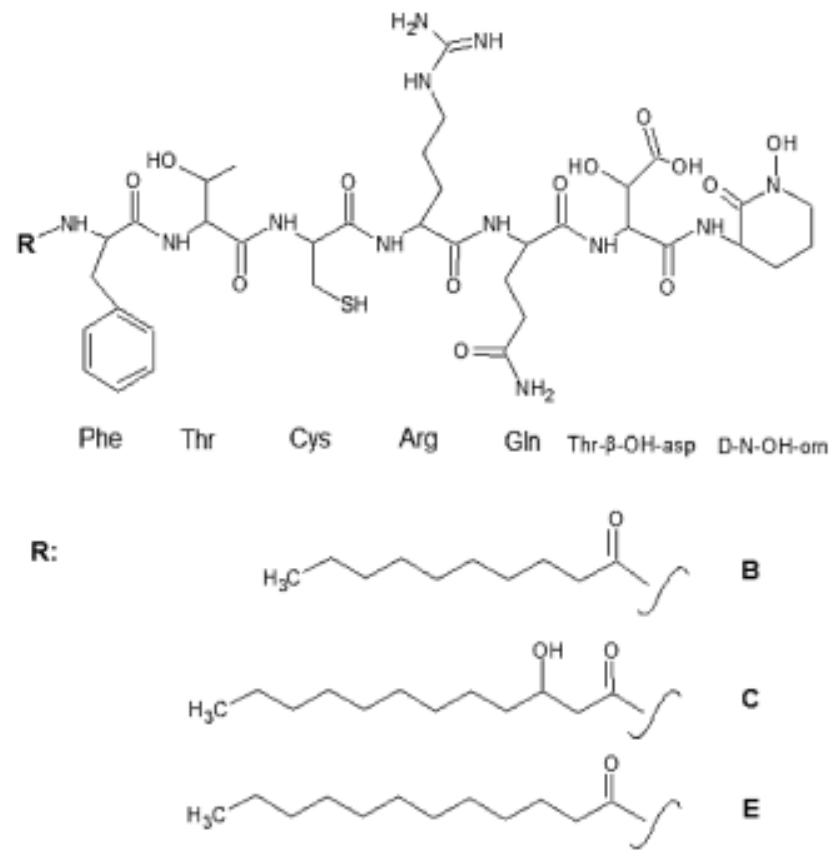

Fig. 2 Molecular structure of halochelins $B, C$ and $E$, produced by Halomonas sp. SL01. On top, the polar head group is presented with the amino acid sequence. On the bottom, the " $R$ " represents the aliphatic tail (fatty acids) attached to it

data. For gas chromatograms and retention times for fatty acid fractions, refer to Online Resources 5 through 8. A detailed and complete siderophore structure is presented in Fig. 2 demonstrating similarities with other marine amphiphilic siderophores, such as the marinobactins.

Halochelin D and F molecular weights were 1079.45 and $1107.48 \mathrm{amu}$, respectively (Online Resources 9 and 10). Parent ions detected by mass spectrometry were attached to hydrogen $(\mathrm{M}+\mathrm{H})$ and hydrated with 5 molecules of water providing the apparent total mass for each molecule (refer to Table 1). The common " $y$ " fragments suggested that, like halochelin B, C and E, the polar head group is conserved. The corresponding amino acid sequence for the polar head group of these amphiphilic siderophore molecules was determined as: proline (Pro), arginine (Arg), serine (Ser), threo- $\beta$-OH-asp (Thr- $\beta$-OH-asp), threonine (Thr), serine (Ser) and $\mathrm{D}-\mathrm{N}-\mathrm{OH}$-ornithine (D-N-OH-orn). The "b" fragmentation pattern was conserved but shifted by $28 \mathrm{amu}$ suggesting differences in an $\mathrm{N}$-terminal hydrophobic tail as seen with the $\mathrm{B}, \mathrm{C}$ and $\mathrm{E}$ family. These fragments also confirmed the amino acid residue sequence. Table 3 shows a summary of fragmentation found with mass spectrometry studies for Halochelin D and F.

As was done with the first suite of halochelins (B, C and E), fragments for each siderophore molecule were compared and mass differences were obtained. Halochelin D total apparent mass was 989 amu and Halochelin F mass was $1017 \mathrm{amu}$. The mass difference between them was 28
Table 3 Mass spectrometry pattern fragmentation of the Halochelins $\mathrm{D}$ and $\mathrm{F}$ produced by Halomonas sp. SL01

\begin{tabular}{lll}
\hline $\begin{array}{l}\text { Fragments } \\
\text { (C-to N-terminus) } \\
\text { y (amu) }\end{array}$ & $\begin{array}{l}D \\
\text { b (amu) }\end{array}$ & $\begin{array}{l}F \\
\text { b (amu) }\end{array}$ \\
\hline $\begin{array}{l}\text { Halochelins } \\
131\end{array}$ & 858 & \\
218 & 771 & 886 \\
319 & 670 & 799 \\
450 & 539 & 698 \\
537 & 452 & 567 \\
693 & 296 & 480 \\
790 & 199 & 324 \\
\hline
\end{tabular}

" $y$ " fragments break at the peptide bond and including the c-terminus. " $b$ " series fragments break the peptide bond and include the n-terminus

amu, which suggested an additional $\left(-\mathrm{CH}_{2} \mathrm{CH}_{2}-\right)$ group in Halochelin F. This 28 amu difference was maintained across the entire "b" series.

To analyze fatty acid tail structure of Halochelin D and F, FAME with gas chromatography (GC) analysis was performed. The $1.9840 \mathrm{~min}$ GC fraction was identified by the Sherlock MIS Software to be the Halochelin D fatty acid (Online Resources 11 and 12). The fraction analysis revealed a fatty acid with structure 12:0 $30 \mathrm{H}$ suggesting that the aliphatic tail was composed of a 12 carbon chain, no double bonds and a hydroxyl $(-\mathrm{OH})$ side group on the third $\alpha$ carbon. Aliphatic tail fragment masses for Halochelin C and D were the same (199 amu) providing the possibility that tail structure was also conserved. For Halochelin F HPLC lyophilized fraction at retention time $2.663 \mathrm{~min}$, a fatty acid with structure 14:0 $30 \mathrm{H}$ was detected (Online Resource 13 and 14). That suggested a saturated fatty acid tail with a 14-carbon chain and a hydroxyl $(-\mathrm{OH})$ side group on the third $\alpha$ carbon. The atomic mass difference between Halochelins D and F was 28 amu, correlating with the findings of MS data. A detailed and complete siderophore structure is presented in Fig. 3 demonstrating similar structures to previously reported amphiphilic siderophores (Martinez and Butler 2007; Vraspir et al. 2011).

\section{Discussion}

The siderophores produced by Halomonas sp. SL01 were found to be amphiphilic and we propose the names of Halochelins B, C, D, E and F. A slight delay in siderophore production relative to cell growth was detected in cultures, suggesting that siderophore production was enhanced as cell growth induced iron limitation. Similar results were presented in a report that studied erythrobactin, a 


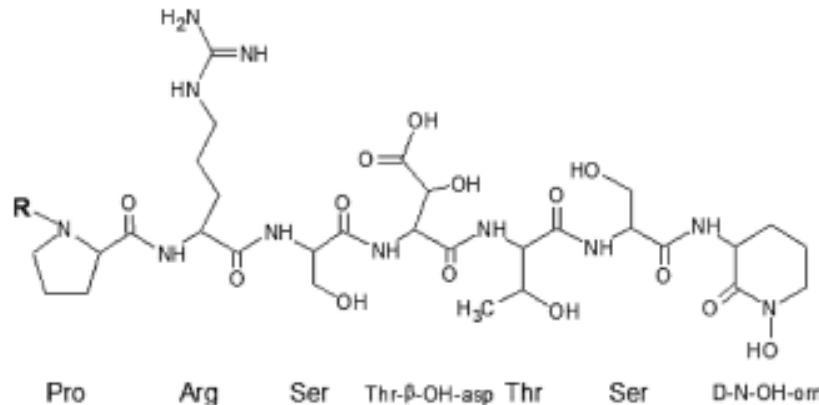

$\mathbf{R}$ :

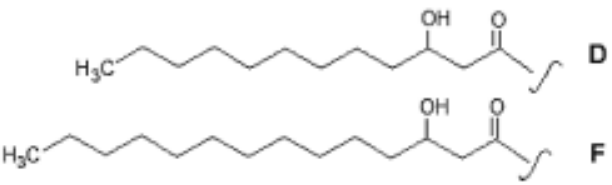

Fig. 3 Halochelins $D$ and $F$, produced by Halomonas sp. SL01. On top, the polar head group is presented with the amino acid sequence. On the bottom, the " $R$ " represents the aliphatic tail (fatty acids) attached to it

hydroxamate-type siderophore produced by the actinomycete S. erythraea (Crosa 2004; Oliveira et al. 2006). Siderophore production was detected after $24 \mathrm{~h}$ of growth. This trend in growth was also found in physiological data about Halomonas sp. SL01 and SL28, in which the microorganisms start to grow and after a time period (usually $24 \mathrm{~h}$ ) produce siderophores. Bertrand and co-workers (2009) also presented data that show similar patterns in siderophore production and microorganism growth at different media $\mathrm{pH}$. The siderophore production delay should be further investigated to determine what cellular processes, at the genetic or molecular levels, controlled it and what ecological or evolutionary importance has. This approach may determine if there are ferritins, bacterioferritins or Dps proteins in Halomonas sp SL01 that may serve as iron reservoirs (Andrews et al. 2003), therefore, explaining siderophore production delay.

Halomonas sp. SL01 was found to produce two different suites of siderophores, each found to contain two distinct amino acid head groups. These amino acid head groups, in addition to the ability to chelate iron, possess polar properties due to the amino acid composition. Fragmentation patterns found in the MS analysis were conserved within each family, suggesting the common headgroup. The presence of proline, phenylalanine and cysteine was surprising, but previous research by other groups has demonstrated siderophores that contain these amino acids: amonabactins (Telford and Raymond 1997), pyochelins (Cox et al. 1981; Liu and Shokrani 1978; Quadri et al. 1999), yersiniabactins (Heesemann et al. 1993; Suo et al. 1999) and thioquinolobactins (Matthijs et al. 2007). The incorporation of serine, arginine, threonine, glutamine, thr- $\beta$-OH-asp and cyclized ornithine is common for amphiphilic siderophores (Dhungana et al. 2007; Homann et al. 2009b; Martinez and Butler 2007; Rosconi et al. 2013; Vraspir et al. 2011). In contrast to the aforementioned studies, our present report describes the first cysteine-, phenylalanine- and proline-containing amphiphilic siderophores produced by a halophile isolated from a soda lake.

As mentioned previously, Halomonas sp. SL01was isolated from Soap Lake, WA (a hypersaline lake). The CAS assay detected siderophore production by the halophile, demonstrating iron binding activity (Schwyn and Neilands 1987). The chrome azurol sulfonate weakly binds ferric iron and transfer of the cation occurs in the presence of high affinity molecules (iron chelating), like siderophores. Mass spectrometry experiments confirmed amphiphilic structure and showed that halochelins are related, but different, to previously characterized siderophores from non-hypersaline/alkaline environments (Martinez and Butler 2007; Vraspir et al. 2011). Because of this affinity of halochelins to ferric iron as demonstrated by the CAS assay (McMillan et al. 2010; Neilands 1976; Schwyn and Neilands 1987; Zheng and Nolan 2012), it is possible that halochelins produced by Halomonas sp. SL01 play a role in the bioavailability of iron within Soap Lake. Soap Lake is a meromictic soda lake, containing three layers, the mixolimnion, chemocline and monimolimnion layers, which are permanently stratified and do not physically mix. Halomonas sp. SL01 was isolated from the upper layer (mixolimnion) which has more exposure to light and oxygen compared to the lower layers. Also, the mixolimnion has a lower dissolved solid concentration (about $14 \mathrm{~g} / \mathrm{L}$ ) compared to the monimolimnion $(140 \mathrm{~g} / \mathrm{L})$, but high when compared to other lake types that intermix their layers (Edmondson and Anderson 1965; Sorokin et al. 2007). Soda lakes dissolved solids values are higher compared to hydrochemical studies of freshwater lakes (Conzonno and Ulibarrena 2010; Karatayev et al. 2008). Karatayev and co-workers measured average dissolved solids at $119 \mathrm{mg} / \mathrm{L}$ in 550 lake in Belarus and Conzonno and Ulibarrena determined the value at $10 \mathrm{~g} / \mathrm{L}$ in Lago Grande, Argentina. These high dissolved solids in soda lakes could provide iron hydroxides that are not soluble in aerobic, alkaline environments (Duckworth et al. 2009) but allowing haloalkaliphilic microorganisms to use siderophores to obtain ferric iron. Evidence of carbon, sulfur and nitrogen biogeochemistry in soda lakes has been described and reviewed (Sorokin et al. 2014); however, information on iron cycling is limited (Emmerich et al. 2012).

Emmerich and co-workers studied the mineralogy, geochemistry and microbial ecology of Lake Kasin (Southern Russia). Ferric oxides content in lake sediments were reported to be $1.13 \%(\mathrm{w} / \mathrm{w})$ and a comparison of ferric reducing microorganisms most probable number (MPN) counts against ferric oxidizing microorganisms values 
revealed similar numbers. However, the research was done on sediments (no pelagic lake waters) and no information on siderophore-mediated iron acquisition was linked to ferric iron cycling. Bacteria and Archaea isolates (from Lake Kasin sediment samples) sequencing data revealed the presence of potential ferric iron-reducing microorganisms, but siderophore-producing organisms were not identified. The marine amphiphilic siderophores, such as the aquachelins, are photoreactive and this may play an important role in biotic and abiotic ferric iron cycling (Barbeau et al. 2001). Photochemical reactivity causes a cleavage on aquachelin, separating it from its fatty acid moiety and reduces ferric iron to ferrous iron. However, the polar head group of aquachelin remains active and continues to facilitate ferric iron chelation. Therefore, photochemical reactivity as an abiotic process likely contributes in ferric iron cycling in pelagic ocean waters providing reduced iron to organisms. How halochelins in pelagic hypersaline lake waters function or contribute to iron cycling is to be investigated, but photoreactivity may play a significant role based on their structural similarity to the aquachelins and other photoreactive amphiphilic siderophores.

Another question lies in the relevance, importance or function that the fatty acyl moieties themselves serve within amphiphilic siderophores. The main hypothesis is that aliphatic tails may provide siderophores the ability to interact with the cell membrane, allowing exposure of the molecule to the extracellular environment without totally releasing the molecule to the extracellular environment. Different diffusion limitation or prevention mechanisms utilized are: (1) acylated siderophores that anchor in the cell membrane; (2) polysaccharide- or matrix-mediated protection of the siderophore in a sheltered environment, limiting siderophore release; or (3) siderophore piracy and utilization of siderophores from various bacteria (Martinez and Butler 2007; Stintzi et al. 2000). Siderophores with small peptide head groups and longer fatty acid tails $(>\mathrm{C} 15)$ would be more likely associated with the cell (Martinez and Butler 2007) as shown with the siderophore marinobactin $F$, which has those characteristics. In the case of marinobactin $\mathrm{F}$, the supernatant concentration of this molecule was low, but halos present (due to the diffusion of the siderophore within the solid medium surrounding bacterial colonies) on inoculated CAS assay plates suggested the possibility that the amphiphile could remain closely associated with the Marinobacter sp cells. Another study presented by the same group shows how marinobactin $\mathrm{E}$ in its deferrated form has more affinity for L- $\alpha$-dimyristoylphosphatidilcholine vesicles than its ferrated counterpart (Xu et al. 2002). It is apparent the importance for the bacterial cell to prevent siderophore diffusion in pelagic ocean waters to optimize iron acquisition in such diluted environments. To confirm this importance in hypersaline lakes, it would be required to assess halochelins membrane interactions and affinity with the corresponding experiments.

In addition to Halomonas sp. SL01, other microorganisms from Bacteria and Archea produce diverse siderophore suites, the composition of which may be in response to different environmental conditions. Valdebenito and coworkers (2006) described how neutral or alkaline media affected siderophore type produced by $E$. coli Nissle 1917 . Enterobactin and aerobactin were produced at higher concentrations in more acidic media compared to salmochelin and yersiniabactin. Pseudomonas aeruginosa produces both pyoverdine and pyochelin siderophores and each one acts in different ways that contribute to pathogenicity. Pyoverdine helps in biofilm formation (Banin et al. 2005); meanwhile, pyochelin helps in host immune response evasion by not binding to siderocalins (proteins that hi-jack siderophores) in mammals (Abergel et al. 2006). In this case, pyochelin still provides iron to Pseudomonas aeruginosa allowing its growth and survival in spite of the host's innate immunity by means of restricting iron. Some bacteria will produce the hydrophilic form of the siderophore but others, due to a change in hydrophilicity, incorporate fatty acids to them (Martin et al. 2006). This will provide an amphiphilicity to the molecule and helps in the organism's survival. Additional studies regarding halochelin synthesis and the distribution of siderophore type produced by SL01 in response to a variety of environmental conditions could provide insight into that organism's adaptions to changing the environmental conditions.

Previous microbiological data in soda lakes, or hypersaline lakes, have shown a diverse microbial population and examples of these environments are Sambhar Lake in India, Soap Lake in WA, USA and Mierlei Lake in Romania (Dirnitriu et al. 2008; Sahay et al. 2012). Similar hypersaline study sites include salt marshes and salterns (Ghozlan et al. 2006). Bacterial phyla observed in these extreme environments are Proteobacteria, Firmicutes and Actinobacteria and different Halomonas-related species (Halomonas campisalis, H. titanicae, H. taeanensis and $H$. elongata among others) have been isolated, sequenced and identified (Sorokin et al. 2014). Halobacteriacea and Methanomicrobia are archaea phyla identified in hypersaline environments and haloarchaeal species (Haloferax volcanii, Halobacterium sp., Halogeometricum borinquense, Haloarcula and Halorubrum alkaliphilum among others) have been isolated, identified and sequenced (Anderson et al. 2011; Ghozlan et al. 2006; Sorokin et al. 2014). Different studies provided information on siderophore-producing microorganisms. Halomonas campisalis is one of the siderophore-producing microorganisms in Sambhar Lake (Sahay et al. 2012) and the presence of siderophore synthesis genes has been described in $H$. borinquense; however, the siderophore structural analyses have been 
performed (Anderson et al. 2011). Another study by Buyer and co-workers (1991) described aerobactin production by a halophilic pseudomonad. Amphiphilic siderophores have been mostly isolated and described from marine environments: marinobactins, aquachelins, ochrobactins, loihichelins, amphibactins and synechobactins (Gauglitz and Butler 2013; Homann et al. 2009a, b; Vraspir et al. 2011). With the present report, it is demonstrated that amphiphilic siderophores are synthesized by microorganisms in other extreme environments, like soda lakes, which differ from those previously identified in marine waters. Structural resemblance is conserved among marine and hypersaline amphiphilic siderophores.

\section{Conclusion}

Prior research to date has suggested the siderophore production potential in hypersaline lakes but until now, no specific identification or structural identification or structural characterization of siderophores from these environments has been made. This report presents and describes one of the first molecular structural characterizations of amphiphilic siderophores (halochelins) produced by a hypersaline lake isolate, Halomonas sp. SL01. These findings present unique amino acid residues in amphiphilic siderophores. Proline, cysteine and phenylalanine amino acid-containing head groups were discovered in these siderophores. The presence of $\alpha$-hydroxycarboxylates and hydroxamates contained within these amino acids could possibly participate as iron-coordinating groups. Future studies should determine the synthesis pathways of these molecules, and examine which and how many, non-ribosomal peptidyl synthetases (NRPSs, responsible for siderophore synthesis) are involved in halochelin synthesis. Determination of the transport of iron into the cells via these siderophores, such as which proteins are involved in siderophore active transport (TonB-like transporter proteins), receptor proteins and periplasmic binding proteins, may yield insight into the specificity of these siderophores for an individual species or show that these siderophores could be universally shared by multiple organisms. Comparisons between synthesis and uptake mechanisms of other species within the genus could provide more details in how the amphiphilic siderophore system evolved in other Halomonas species and describe its ecological role in diverse environments.

\footnotetext{
Acknowledgments Mass spectrometry data was done with the assistance of Dr. Jonathan Hilmer from the Mass Spectrometry Facility in the Department of Chemistry and Biochemistry, Montana State University-Bozeman. Special thanks to Ann Willis for HPLC support. Also thanks to Dr. Royce Wilkinson for assistance with the lyophilizer and to MIDI Labs, Inc. for the FAME and GC analysis. Funding was from the National Science Foundation (NSF) Grant Number EEC-0927109.
}

\section{Compliance with ethical standards}

Conflict of interest The authors declare that they have no conflict of interest.

\section{References}

Abergel RJ, Wilson MK, Arceneaux JEL, Hoette TM, Strong RK, Byers BR, Raymond KN (2006) Anthrax pathogen evades the mammalian immune system through stealth siderophore production. Proc Natl Acad Sci USA 103:18499-18503. doi:10.1073/ pnas. 0607055103

Amin SA, Green DH, Al Waheeb D, Gaerdes A, Carrano CJ (2012) Iron transport in the genus Marinobacter. Biometals 25:135-147. doi:10.1007/s10534-011-9491-9

Anderson I et al (2011) Novel insights into the diversity of catabolic metabolism from ten haloarchaeal genomes. PLoS One 6:12. doi:10.1371/journal.pone.0020237

Andrews SC, Robinson AK, Rodriguez-Quinones F (2003) Bacterial iron homeostasis. FEMS Microbiol Rev 27:215-237. doi:10.1016/s0168-6445(03)00055-x

Banin E, Vasil ML, Greenberg EP (2005) Iron and Pseudomonas aeruginosa biofilm formation. Proc Natl Acad Sci USA 102:1107611081. doi:10.1073/pnas.0504266102

Barbeau K, Rue EL, Bruland KW, Butler A (2001) Photochemical cycling of iron in the surface ocean mediated by microbial iron(III)-binding ligands. Nat 413:409-413. doi: $10.1038 / 35096545$

Butler A, Theisen RM (2010) Iron(III)-siderophore coordination chemistry: reactivity of marine siderophores. Coord Chem Rev 254:288-296. doi:10.1016/j.ccr.2009.09.010

Conzonno VH, Ulibarrena J (2010) Hydrochemistry of Lakes of the Patagonian Province of Tierra del Fuego (Argentina). Env Earth Sci 59:1431-1436. doi:10.1007/s12665-009-0129-y

Cox C, Rinehart K, Moore M, Cook J (1981) Pyochelin - Novel Structure of an Iron-Chelating Growth Promoter for PseudomonasAeruginosa. Proc Natl Acad Sci USA-Biol Sci 78:4256-4260. doi: 10.1073/pnas.78.7.4256

Crosa JH (2004) Iron transport in bacteria, 1st edn. ASM Press, Washington, DC

Dhungana $S$ et al (2007) Purification and characterization of thodobactin: a mixed ligand siderophore from Rhodococcus rhodochrous strain OFS. Biometals 20:853-867. doi:10.1007/ s10534-006-9079-y

Dirnitriu PA, Pinkart HC, Peyton BM, Mormile MR (2008) Spatial and temporal patterns in the microbial diversity of a meromictic soda lake in Washington State. Appl Env Microbiol 74:48774888. doi:10.1128/aem.00455-08

Duckworth OW, Holmstrom SJM, Pena J, Sposito G (2009) Biogeochemistry of iron oxidation in a circumneutral freshwater habitat. Chem Geol 260:149-158. doi:10.1016/j.chemgeo.2008.08.027

Edmondson WT, Anderson GC (1965) Some features of saline lakes in Central Washington. Limnol Oceanogr 10:R87-R96

Emmerich M, Bhansali A, Loesekann-Behrens T, Schroeder C, Kappler A, Behrens S (2012) Abundance, distribution, and activity of fe(II)-oxidizing and fe(III)-reducing microorganisms in hypersaline sediments of Lake Kasin, Southern Russia. Appl Env Microbiol 78:4386-4399. doi:10.1128/aem.07637-11

Gauglitz JM, Butler A (2013) Amino acid variability in the peptide composition of a suite of amphiphilic peptide siderophores from an open ocean Vibrio species. J Biol Inorg Chem 18:489-497. doi: $10.1007 / \mathrm{s} 00775-013-0995-3$

Gauglitz JM, Zhou HJ, Butler A (2012) A suite of citrate-derived siderophores from a marine Vibrio species isolated following 
the Deepwater Horizon oil spill. J Inorg Biochem 107:90-95. doi:10.1016/j.jinorgbio.2011.10.013

Ghozlan H, Deif H, Abu Kandil R, Sabry S (2006) Biodiversity of moderately halophilic bacteria in hypersaline habitats in Egypt. J Gen Appl Microbiol 52:63-72. doi:10.2323/jgam.52.63

Heesemann J, Hantke K, Vocke T, Saken E, Rakin A, Stojiljkovic I, Berner R (1993) Virulence of yersinia-enterocolitica is closely associated with siderophore production, expression of an iron-repressible outer-membrane polypeptide of 65000 da and pesticin sensitivity. Molec Microbiol 8:397-408. doi:10.1111/j.1365-2958.1993.tb01583.x

Homann V, Edwards K, Webb E, Butler A (2009a) Siderophores of Marinobacter aquaeolei: petrobactin and its sulfonated derivatives. Biometals 22:565-571. doi:10.1007/s10534-009-9237-0

Homann V, Sandy M, Tincu J, Templeton A, Tebo B, Butler A (2009b) Loihichelins A-F, a Suite of Amphiphilic Siderophores Produced by the Marine Bacterium Halomonas LOB-5. J Nat Prod 72:884-888. doi: 10.1021/np800640h

Ito Y, Butler A (2005) Structure of synechobactins, new siderophores of the marine cyanobacterium Synechococcus sp PCC 7002. Limnol Oceanogr 50:1918-1923

Karatayev AY, Burlakova LE, Dodson SI (2008) Community analysis of Belarusian lakes: correlations of species diversity with hydrochemistry. Hydrobiol 605:99-112. doi:10.1007/ s10750-008-9323-2

Liu P, Shokrani F (1978) Biological-Activities of Pyochelins - IronChelating Agents of Pseudomonas-Aeruginosa. Infect Immun 22:878-890

Martin J, Ito Y, Homann V, Haygood M, Butler A (2006) Structure and membrane affinity of new amphiphilic siderophores produced by Ochrobactrum sp SP18. J Biol Inorg Chem 11:633641. doi:10.1007/s00775-006-0112-y

Martinez J, Butler A (2007) Marine amphiphilic siderophores: marinobactin structure, uptake, and microbial partitioning. J Inorg Biochem 101:1692-1698. doi:10.1016/j.jinorgbio.2007.07.007

Matthijs S, Tehrani K, Laus G, Jackson R, Cooper R, Cornelis P (2007) Thioquinolobactin, a Pseudomonas siderophore with antifungal and anti-Pythium activity. Environ Microbiol 9:425-434. doi:10.1111/j.1462-2920.2006.01154.x

McMillan DGG et al (2010) Acquisition of Iron by Alkaliphilic Bacillus Species. Appl Environ Microbiol 76:6955-6961. doi:10.1128/aem.01393-10

Neilands JB (1976) Siderophores: diverse roles in microbial and human physiology. Ciba Found Symp (51):107-124

Oliveira PH, Batagov A, Ward J, Baganz F, Krabben P (2006) Identification of erythrobactin, a hydroxamate-type siderophore produced by Saccharopolyspora erythraea. Lett Appl Microbiol 42:375-380. doi:10.1111/j.1472-765X.2006.01849.X

Pattus F, Abdallah M (2000) Siderophores and iron-transport in microorganisms. J Chin Chem Soc 47:1-20

Pollock J, Weber KA, Lack J, Achenbach LA, Mormile MR, Coates JD (2007) Alkaline iron(III) reduction by a novel alkaliphilic, halotolerant, Bacillus sp isolated from salt flat sediments of Soap Lake. Appl Microbiol Biotechnol 77:927-934. doi:10.1007/ s00253-007-1220-5

Quadri LEN, Keating TA, Patel HM, Walsh CT (1999) Assembly of the Pseudomonas aeruginosa nonribosomal peptide siderophore pyochelin: in vitro reconstitution of aryl-4,2-bisthiazoline synthetase activity from PchD, PPchE, and PchF. Biochem 38:14941-14954. doi:10.1021/bi991787c

Raymond KN, Dertz EA (2004) Biochemical and physical properties of siderophores. Iron transport in bacteria, vol 1. First edn. ASM Press, Washington, DC, pp 3-17
Rosconi F et al (2013) Identification and structural characterization of serobactins, a suite of lipopeptide siderophores produced by the grass endophyte Herbaspirillum seropedicae. Environ Microbiol 15:916-927. doi:10.1111/1462-2920.12075

Sahay H, Mahfooz S, Singh AK, Singh S, Kaushik R, Saxena AK, Arora DK (2012) Exploration and characterization of agriculturally and industrially important haloalkaliphilic bacteria from environmental samples of hypersaline Sambhar lake, India. World J Microbiol Biotechnol 28:3207-3217. doi:10.1007/ s11274-012-1131-1

Sandy M, Butler A (2009) Microbial Iron Acquisition: marine and Terrestrial Siderophores. Chem Rev 109:4580-4595. doi: $10.1021 / \mathrm{cr} 9002787$

Sandy M, Han A, Blunt J, Munro M, Haygood M, Butler A (2010) Vanchrobactin and Anguibactin Siderophores Produced by Vibrio sp DS40M4. J Nat Prod 73:1038-1043. doi:10.1021/ np900750g

Schwyn B, Neilands JB (1987) Universal Chemical-Assay for the Detection and Determination of Siderophores. Anal Biochem 160:47-56. doi:10.1016/0003-2697(87)90612-9

Sorokin DY, Foti M, Pinkart HC, Muyzer G (2007) Sulfur-oxidizing bacteria in Soap Lake (Washington State), a meromictic, haloalkaline lake with an unprecedented high sulfide content. Appl Environ Microbiol 73:451-455. doi:10.1128/aem.02087-06

Sorokin DY, Berben T, Melton ED, Overmars L, Vavourakis CD, Muyzer G (2014) Microbial diversity and biogeochemical cycling in soda lakes. Extrem 18:791-809. doi:10.1007/ s00792-014-0670-9

Stintzi A, Barnes C, Xu L, Raymond KN (2000) Microbial iron transport via a siderophore shuttle: a membrane ion transport paradigm. Proc Natl Acad Sci USA 97:10691-10696. doi:10.1073/ pnas. 200318797

Suo Z, Walsh CT, Miller DA (1999) Tandem heterocyclization activity of the multidomain $230 \mathrm{kDa}$ HMWP2 subunit of Yersinia pestis yersiniabactin synthetase: interaction of the 1-1382 and 1383-2035 fragments. Biochem 38:14023-14035. doi:10.1021/ bi $991574 n$

Telford JR, Raymond KN (1997) Amonabactin: a family of novel siderophores from a pathogenic bacterium. J Biol Inorg Chem 2:750-761. doi:10.1007/s007750050191

Valdebenito M, Crumbliss AL, Winkelmann G, Hantke K (2006) Environmental factors influence the production of enterobactin, salmochelin, aerobactin, and yersiniabactin in Escherichia coli strain Nissle 1917. Internatl J Med Microbiol 296:513-520. doi:10.1016/j.ijmm.2006.06.003

VanEngelen MR, Peyton BM, Mormile MR, Pinkart HC (2008) $\mathrm{Fe}(\mathrm{III}), \mathrm{Cr}$ (VI), and $\mathrm{Fe}$ (III) mediated $\mathrm{Cr}$ (VI) reduction in alkaline media using a Halomonas isolate from Soap Lake, Washington. Biodegrad 19:841-850. doi:10.1007/s10532-008-9187-1

Vraspir JM, Holt PD, Butler A (2011) Identification of new members within suites of amphiphilic marine siderophores. Biometals 24:85-92. doi:10.1007/s10534-010-9378-1

Xu G, Martinez J, Groves J, Butler A (2002) Membrane affinity of the amphiphilic marinobactin siderophores. J Amer Chem Soc 124:13408-13415. doi:10.1021/ja026768w

Zheng TF, Nolan EM (2012) Siderophore-based detection of Fe(III) and microbial pathogens. Metallomics 4:866-880. doi:10.1039/ c2mt20082a 